\title{
Robustness of the avalanche dynamics in data packet transport on scale-free networks
}

\author{
E. J. Lee, K.-I. Goh, B. Kahng, and D. Kim \\ School of Physics and Center for Theoretical Physics, \\ Seoul National University NS50, Seoul 151-747, Korea
}

(Dated: November 8, 2018)

\begin{abstract}
We study the avalanche dynamics in the data packet transport on scale-free networks through a simple model. In the model, each vertex is assigned a capacity proportional to the load with the proportionality constant $1+a$. When the system is perturbed by a single vertex removal, the load of each vertex is redistributed, followed by subsequent failures of overloaded vertices. The avalanche size depends on the parameter $a$ as well as which vertex triggers it. We find that there exists a critical value $a_{c}$ at which the avalanche size distribution follows a power law. The critical exponent associated with it appears to be robust as long as the degree exponent is between 2 and 3 , and is close in value to that of the distribution of the diameter changes by single vertex removal.

PACS numbers: 89.70.+c, 89.75.-k, 05.10.-a
\end{abstract}

Avalanche dynamics, triggered by small initial perturbation, but spreading to other constituents successively, is one of intriguing problems in physics [1, 2, 3, 4, 5. 6, 7, 8, 9, 10, 11]. Such avalanche dynamics manifests itself in diverse forms such as cultural fads 1], virus spreading [2], disease contagion [3], blackout in power transmission grids [4, 5], data packet congestion in the Internet [6, 7], and so on. In particular, the avalanche phenomena on complex networks are interesting, because they occur more frequently and their impact can be more severe than those occurring in the Euclidean space due to the close inter-connectivity among constituents in complex networks.

To understand the intrinsic nature of the avalanche dynamics on complex networks, the sandpile model proposed by Bak, Tang, and Wiesenfeld has been studied on scale-free (SF) networks recently 12. The SF network is the network whose degree distribution follows a power law, $p_{d}(k) \sim k^{-\gamma}$. Since the sandpile model is a selforganized critical model, the avalanche size distribution follows a power law, $p_{a}(s) \sim s^{-\tau}$, where $s$ is the avalanche size. In the sandpile model, the exponent $\tau$ depends on the degree exponent $\gamma$ of the embedded SF network as $\tau_{\mathrm{BTW}}=\gamma /(\gamma-1)$ for $2<\gamma<3$ when the toppling threshold of each vertex is equal to its degree. However, when the toppling threshold is fixed as a constant, independent of degree, the exponent $\tau_{\mathrm{MF}}=3 / 2$, being equal to the mean field value in the Euclidean space. Thus, it would be interesting to find an example of avalanche dynamics where the avalanche size distribution follows a power law with a nontrivial exponent, but different from the mean field value, and robust against variation of degree exponents. For this purpose, in this paper, we study the model proposed by Motter and Lai (ML) 7], designed to exploit the avalanche dynamics in the process of data packet transport on complex networks.

In the ML model, each vertex is assigned a finite capacity, given as

$$
c_{j}=(1+a) \ell_{j}^{(0)}
$$

where $a$ is a control parameter and $\ell_{j}^{(0)}$ is the load of ver- tex $j$. The load of a given vertex is defined as the sum of the effective number of data packets passing through that vertex when every pair of vertices send and receive a unit data packet. The data packets are allowed to travel along the shortest pathways between a given pair of vertices and are divided evenly at each branching point [13, 14]. For SF networks, the load of each vertex is heterogeneous, and its distribution also follows a power law, $p_{\ell}(\ell) \sim \ell^{-\delta}$. The superscript (0) in Eq. (1) indicates the load without any removal of vertices. The excess term $a \ell_{j}^{(0)}$ in Eq. (1) provides the ability to tolerate the additional burden and may describe the excess buffer at the routers in the Internet, for example. The basic assumption of the ML model is that the size of such an excess buffer is proportional to the activity at the vertex, the load $\ell_{j}^{(0)}$. The control parameter $a$ sets the global level of tolerance of the system.

Next, we remove a vertex $i$ intentionally, which we call triggering vertex. Then each pair of remaining vertices whose shortest pathway had passed through that triggering vertex should find detours, resulting in rearrangement of the shortest pathways over the network, and the load at a remaining vertex $j$ takes a new value, which is denoted as $\ell_{j}^{(i)}$. If the load $\ell_{j}^{(i)}$ exceeds its capacity $c_{j}$ given by Eq. (11), then the vertex $j$ would fail irreversibly. Other overloaded vertices also fail at the same time. These are the failures by the first shock, marked green with the symbol $(\diamond)$ in Fig. 1. After then, the shortest pathway configurations would rearrange again, and the overloaded vertices fail successively until no overloaded vertices remain. The avalanche size $s_{i}$ is defined as the total number of failed vertices throughout the whole process of the avalanche triggered by the vertex $i$. Note that in this model, failures do not necessarily proceed contiguously, that is, through the neighbors of vertices previously failed, but spread over the entire system through nonlocal dynamics as shown in Fig. 1. For such nonlocal dynamics, the branching process formalism cannot be used to obtain the avalanche size distribution of the ML model. 


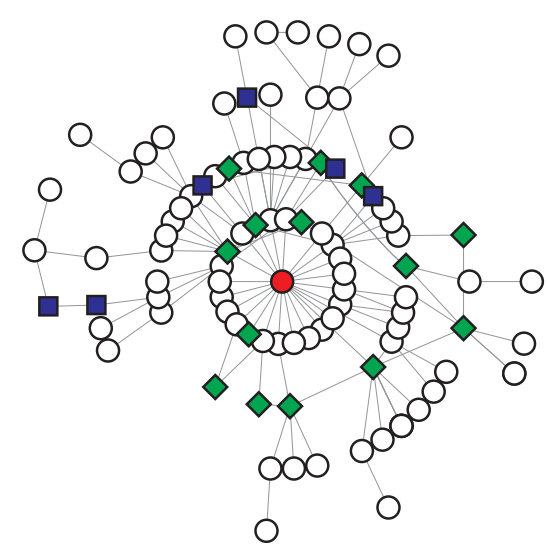

FIG. 1: (Color online) Plot of the avalanche dynamics pattern at $a_{c}=0.15$ for a given small-size network. Cascading failures starting from the central vertex spread in a nonlocal way following the steps, $\bigcirc$ (red), $\diamond$ (green), and $\square$ (blue).

In the original work, ML measured the ratio $G_{i}=$ $N_{i}^{\prime} / N$, where $N$ and $N_{i}^{\prime}$ are the numbers of vertices before and after cascading failures, respectively, when the triggering vertex is $i$. Note that the avalanche size corresponds to $s_{i}=N-N_{i}^{\prime}$. ML found that $G_{i}$ depends on the degree $k_{i}$ of the triggering vertex $i$ as well as the control parameter $a$. When $a$ is large (small), the capacity of each vertex is large (small), so that the number of failed vertices is small (large) and $G_{i}$ is close to one (zero). Moreover, when the degree of the triggering vertex is large (small), $G_{i}$ is close to zero (one), and the system is vulnerable (robust). Such numerical results suggest that there may occur a phase transition in the avalanche size. In this paper, we find numerically that indeed there exists a critical value $a_{c}$ at which the avalanche size distribution follows a power law, $p_{a}(s) \sim s^{-\tau}$. We also study various features of the avalanche dynamics at the critical point.

Let us first investigate the distribution of $\left\{s_{i}\right\}$, the avalanche size distribution $p_{a}(s)$. For large (small) $a$, the number of overloaded vertices is small (large), so that the avalanche size is finite (diverges) and the system may be considered as in a subcritical (supercritical) phase. We find that there exists a characteristic value $a_{c}$ between the two regimes, where the avalanche size distribution follows a power law, $p_{a}(s) \sim s^{-\tau}$ as shown in Fig. 2] Numerical simulations are performed for the Barabási-Albert (BA) model 15] with different degree exponent values. We find that $a_{c} \approx 0.15$, and $\tau \approx 2.1(1)$, both of which are likely to be robust for different degree exponents $\gamma$ as long as $2<\gamma<3$. While it is not manifest why such a robust behavior occurs in the avalanche size distribution, it is noteworthy to remind that other problems related to the shortest pathways such as the load distribution and the diameter change distribution are also likely to be robust. Thus the robustness of the avalanche size distribution may be caused by the notion of the shortest pathway. For $\gamma>3$, however, $p_{a}(s)$ decays with exponent larger than

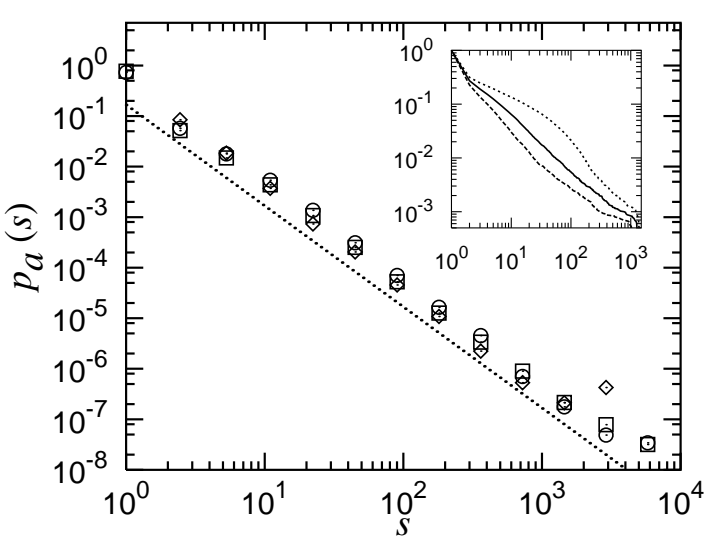

FIG. 2: Plot of the avalanche size distribution for the BA model at $a_{c}=0.15$ with different $\gamma=3.0(\bigcirc), 2.6(\square)$, and $2.2(\diamond)$. The mean degree is 4 , and the system size is $N=$ $10^{4}$. The dotted line has a slope -2.1 , drawn for reference. The avalanche size distributions are obtained by deleting each vertex $i$ in turn and measuring the respective avalanche size $s_{i}$, then tabulating the histogram of $s_{i}$, normalized by the number of triggering vertices $N$. Inset: the avalanche size distribution(cumulative) under the same condition for $\gamma=$ 3.0, but with $a=0.11$ (top), 0.15 (middle), and 0.2 (bottom).

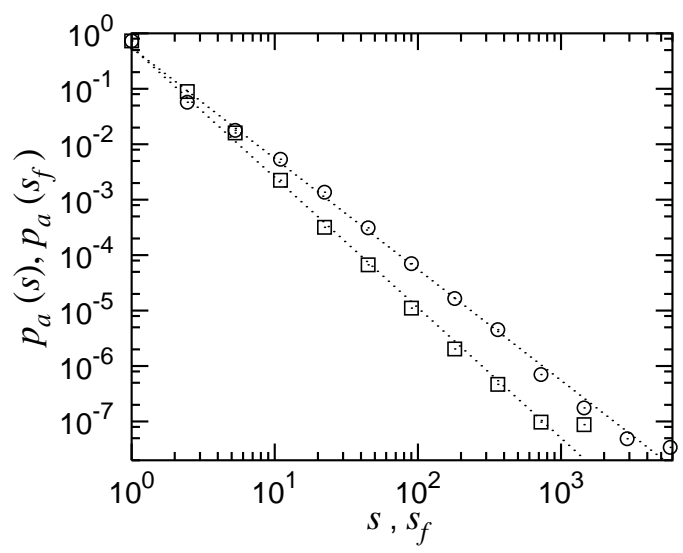

FIG. 3: Plot of the avalanche size distribution for the BA model with $\gamma=3$ by the first shock $(\square)$, compared with the avalanche size distribution including the entire process $(\bigcirc)$. The slopes of dotted and dashed lines are -2.3 and -2.1 , respectively, drawn for reference.

$\tau \approx 2.1$ or exponentially depending on $\gamma$. The avalanche size distribution by the first shock behaves differently as $p_{a}\left(s_{f}\right) \sim s_{f}^{-2.3}$, which is shown in Fig. 3. We also check the avalanche size distribution for real world networks. For the yeast protein interaction network and the Internet, we obtain $\tau \approx 2.3(1)$ and $\tau \approx 1.8(1)$, respectively, as shown in Fig. [4 Note that the degree exponent of the yeast protein interaction network is $\gamma \approx 3.4[16]$, slightly larger than 3 , thus the exponent $\tau \approx 2.3$ is somewhat larger than 2.1(1) obtained in the BA model for $2<\gamma<3$.

The deviation of the exponent $\tau$ for the Internet is 


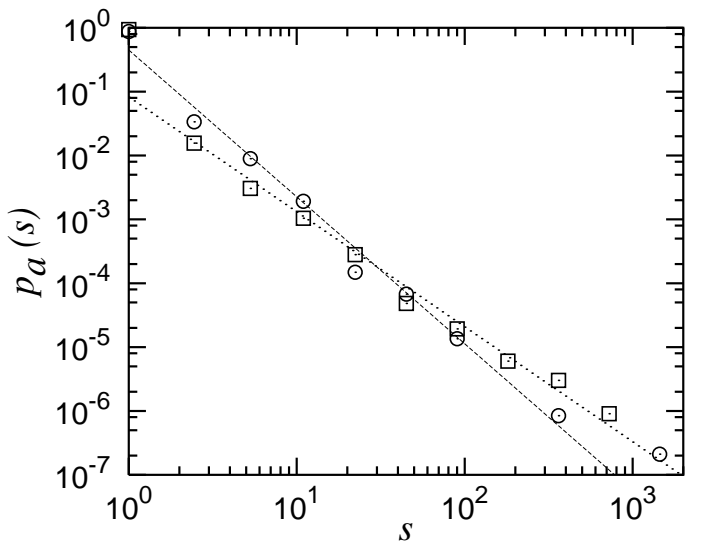

FIG. 4: Plot of the avalanche size distribution for the yeast protein interaction network $(\bigcirc)$ and the Internet $(\square)$ at $a_{c}=$ 0.15 . The slopes of dotted and dashed lines are -1.8 and -2.3 , respectively, drawn for reference.

rooted from its different network structure from those of the protein interaction networks or the BA-type model networks: it was found through recent several studies that the Internet structure is effectively tree-like, while the protein interaction network and BA-type model networks contain diverse connections 14, 17]. Accordingly, when a vertex on a branch of tree structure is removed, the giant cluster is divided into two or more components, and the giant cluster size shrinks apparently. Such a case occurs more often in the Internet than in other-type networks, because the Internet is tree-like. Due to this fact, the avalanche size statistics of the Internet is different from that of other networks. On the other hand, while the rule of the ML model may not be relevant to the dynamics in the protein interaction network or cellular networks, cascading failure occurring in cellular networks is an important concept. For example, the protein interaction network provides the basic operational protocol in various signal transduction and functional pathways. In such a system, when a certain element (a protein or a substrate) fails or is removed (perturbed), others should take over its burden to survive the lack thereof, although the mechanism by which the cascade spreads could be different from that of the ML model. Studies in this direction have recently been carried out for the metabolic networks 18, 19.

Next, we examine the relationship of the mean avalanche size, denoted by $\langle s\rangle\left(k_{\mathrm{del}}\right)$, over different triggering vertices but with a given degree $k_{\text {del }}$ at $a_{c}$ in Fig. [5 We find that the quantity $\langle s\rangle\left(k_{\mathrm{del}}\right)$ increases with increasing $k_{\text {del }}$. However, there occur large fluctuations in $\langle s\rangle\left(k_{\text {del }}\right)$, in particular, for small $k_{\text {del }}$. Note that if the orderings in the magnitude of $\langle s\rangle\left(k_{\mathrm{del}}\right)$ and $k_{\text {del }}$ are preserved, $p_{a}(s) \mathrm{d} s=p_{d}(k) \mathrm{d} k$ and hence one has the relation $\langle s\rangle\left(k_{\text {del }}\right) \sim k_{\text {del }}^{(\gamma-1) /(\tau-1)}$. Indeed, Fig. 5 exhibits such a behavior. To examine the fluctuations of $\langle s\rangle\left(k_{\text {del }}\right)$ for given $k_{\text {del }}$, we consider the distribution function $q(x)$

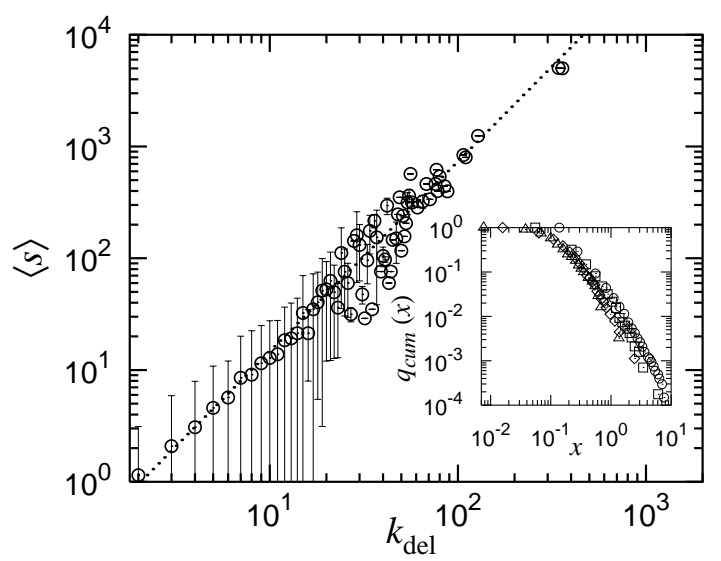

FIG. 5: Plot of the mean avalanche size $\langle s\rangle$ versus the degree of the triggering vertex $k_{\text {del }}$ for the BA model $(\gamma=3)$ at $a_{c}$. Data points $(\bigcirc)$ are averaged over different avalanche sizes triggered by the vertices with a given $k_{\text {del }}$. The standard deviation of each data point is represented by a bar. The slope of the dotted line is the theoretical value 1.8, drawn for reference. Inset: Cumulative plot of the avalanche size distribution with the rescaled quantity $x=s / k_{\mathrm{del}}^{(\gamma-1) /(\tau-1)}$ for $k_{\mathrm{del}}=3(\circ), 5(\square), 10(\diamond)$, and $15(\triangle)$.

of the avalanche sizes for given $k_{\text {del }}$ with a rescaled quantity, $x=s / k_{\text {del }}^{(\gamma-1) /(\tau-1)}$. Shown in the inset of Fig. [5]are the data of the cumulative distribution of $q_{\text {cum }}(x)$ for different $k_{\text {del }}$, which collapse onto a single curve exhibiting a fat-tail behavior as $q(x) \sim x^{-3.2}$ for large $x$.

Next, to study how much a given vertex with degree $k$ is vulnerable or robust under a random vertex failure, we count the number of failures $n_{j}$ of a vertex $j$ out of $N$ cascading events when each of $N$ vertices acts as the triggering vertex. At this point, it is convenient to consider the random variables $x_{j}{ }^{i}$ which take the value 1 if the vertex $j$ topples due to the triggering vertex $i$ and 0 otherwise. In terms of $x_{j}{ }^{i}, \sum_{j} x_{j}{ }^{i}=s_{i}$ and $\sum_{i} x_{j}{ }^{i}=n_{j}$. Let $f(k)$ be the average of $n_{j} / N$ over the vertices with degree $k$. Fig. [6] shows the function $f(k)$ versus $k$. It increases with increasing $k$ for small $k$ and exhibits a peak in the intermediate range of $k$. For large $k, f(k)$ is almost independent of $k$. This result implies that the vertices with degree in the intermediate range are more vulnerable. Meanwhile, we note that the asymptotic value of $f(k)$ for large $k$ is $\sim \mathcal{O}(1 / N)$. This is because a vertex with large $k$ hardly fails through the cascading failure process triggered by other vertices, but fails trivially when itself acts as the triggering vertex. The peak at the intermediate value of $k$ in $f(k)$ can be understood in the following heuristic way. The load itself quantifies the level of traffic coming through the vertex, so we can expect that the higher the load is, the more excess traffic it would get by the breakdown of other vertices. On the other hand, since the excess capacity $a \ell_{j}^{(0)}$ is assigned in a multiplicative way, the higher the load is, the larger room to accommodate the excess is, reducing the occa- 


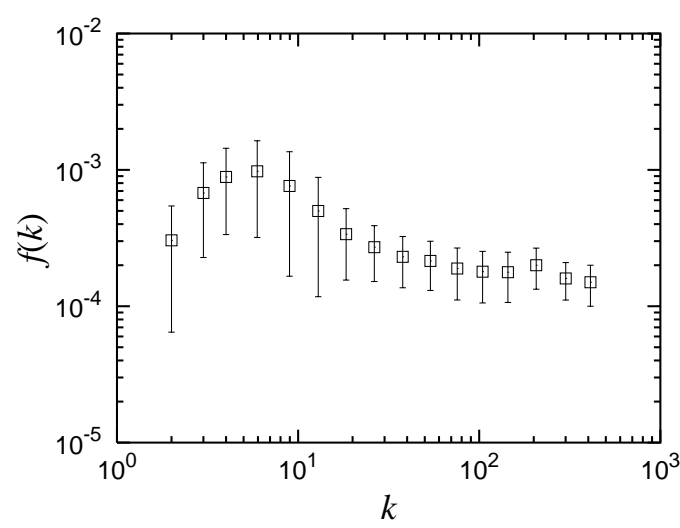

FIG. 6: Plot of the failure fraction $f$ versus degree $k$ at $a_{c}$ for the BA model $(\gamma=3)$ with $N=10^{4}$. Data points are logarithmically binned. Error bars represent the standard deviations for each bin.

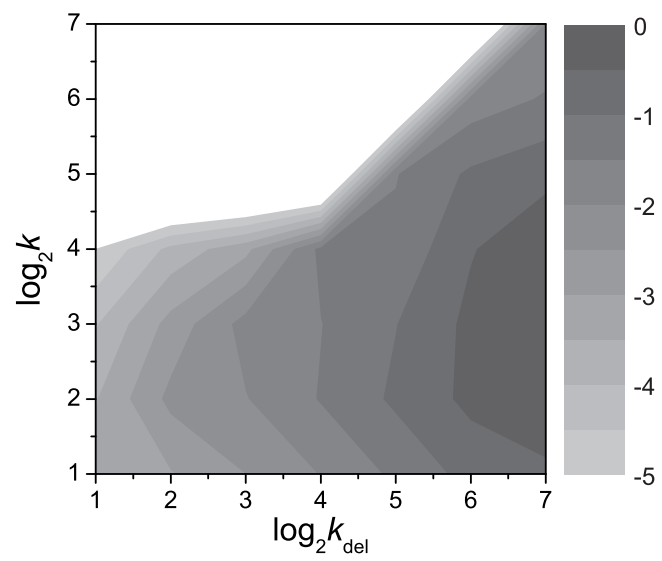

FIG. 7: Plot of the logarithm (with base 10) of the failure correlation function $c\left(k, k_{\mathrm{del}}\right)$ as functions of the degrees of the failed vertex $k$ and of the triggering vertex $k_{\text {del }}$. Data are logarithmically binned to reduce fluctuations. Simulation is performed for the BA model with $\gamma=3, N=3000$ and averaged over 10 configurations.

sion to be overloaded. These two factors compete each other, generating a peak in the intermediate range of $k$ in $f(k)$. That implies the vertices with intermediate degrees are more vulnerable. Such a behavior can also be seen in the information cascade model of Watts 1].

This result is also reminiscent of the avalanche dynamics of the sandpile model. The hubs, vertices with large degrees, play a role of the reservoir against failures [12]. We also consider the failure correlation function $c\left(k, k_{\mathrm{del}}\right)$, defined as the average of $x_{j}{ }^{i}$ with the constraints $k_{j}=k$ and $k_{i}=k_{\mathrm{del}}, k_{i}$ denoting the degree of a vertex $i$. The darkest region in the bottom-right corner of Fig. 7 indicates that vertices with small degrees easily fail by the trigger of vertices with large degrees, whereas the reverse rarely happen, particularly for large $k_{\mathrm{del}}$, as manifested by the white region in the upper-left part of Fig. 7.

It is interesting to notice that the avalanche size distribution behaves similarly to the diameter change distribution [20]. Diameter is the average number of hops between every pair of vertices. Let $d^{(0)}$ be the diameter of a given network, where the superscript (0) means unperturbed network. When the network is perturbed by the removal of a vertex $i$, the diameter changes accordingly, and the diameter of the remaining network is denoted as $d^{(i)}$. Then the dimensionless quantity $\Delta_{i}=\left(d^{(i)}-d^{(0)}\right) / d^{(0)}$ is measured for all $i$, and then its distribution function, composed of $\left\{\Delta_{i}\right\}$, behaves as $p_{\text {DC }}(\Delta) \sim \Delta^{-\zeta}$ for large $\Delta$. The exponent $\zeta$ was measured to be $\zeta \approx 2.2(1)$ for most artificial SF networks including the $\mathrm{BA}$ model, insensitive to the degree exponent $\gamma$ as long as $2<\gamma<3$, and $\zeta \approx 2.3(1)$ for the yeast protein interaction network, but $\zeta \approx 1.7(1)$ for the Internet. All the above values of the exponent $\zeta$ are close to corresponding values of $\tau$ for the avalanche size distribution of the ML model. In addition, the exponents $\tau$ and $\zeta$ are also close in values to the load distribution exponent $\delta$ except for a few examples such as the Internet. Thus, it would be interesting to investigate the origin of such coincidences on a fundamental level.

Finally, it is noteworthy that recently Zhao et al. [21] also studied the phase transition of the cascading failure for the ML model. They estimated the critical point to be $a_{c} \approx 0.1$ by comparing the load distribution before and after the deletion of the hub. Their estimation is not inconsistent with our numerical estimation. However, the avalanche size distribution studied in this work provides a better criterion for the phase transition point.

In conclusion, we have studied the avalanche dynamics in the model proposed by Motter and Lai, describing the data packet transport on SF networks. Depending on the model parameter $a$, which controls the magnitude of the capacity of each vertex, the pattern of avalanche dynamics can change. For small $a$, cascading failure spreads over the entire system, corresponding to supercritical behavior in avalanche dynamics. While, for large $a$, cascading failure is confined in a small region, and avalanche size follows a subcritical behavior. At the critical point $a_{c}$, the avalanche size distribution follows a power law with exponent $\tau$. The exponent $\tau$ seems to be robust for different degree exponent $\gamma$ as long as $2<\gamma<3$, and is likely to be close to the exponent of the diameter change distribution.

This work is supported by the KOSEF grants No. R142002-059-01000-0 in the ABRL program. 
86, 3200 (2001); Phys. Rev. E 63, 066117 (2001); ibid 65, 035108 (2002).

[3] M. E. J. Newman, Phys. Rev. E 66, 016128 (2002).

[4] M. L. Sachtjen, B. A. Carreras, and V. E. Lynch, Phys. Rev. E 61, 4877 (2000).

[5] R. Kinney, P. Crucitti, R. Albert, and V. Latora, cond-mat/0410318

[6] P. Holme and B. J. Kim, Phys. Rev. E 65, 066109 (2002).

[7] A.E. Motter and Y.-C. Lai, Phys. Rev. E 66, 065102(R)(2002).

[8] Y. Moreno, R. Pastor-Satorras, A. Vazquez and A. Vespignani, Europhys. Lett. 62, 292 (2003).

[9] P. Crucitti, V. Latora, and M. Marchiori, Phys. Rev. E 69, 045104(R) (2004).

[10] G. Bianconi and M. Marsili, Phys. Rev. E 70, 035105(R) (2004).

[11] Y. Moreno, J. B. Gómez, and A. F. Pacheco, Europhys. Lett. 58, 630 (2002).

[12] K.-I. Goh, D.-S. Lee, B. Kahng, and D. Kim, Phys. Rev.
Lett. 91, 148701 (2003).

[13] K.-I. Goh, B. Kahng, and D. Kim, Phys. Rev. Lett. 87, 278701 (2001).

[14] K.-I. Goh, E. Oh, H. Jeong, B. Kahng, and D. Kim, Proc. Natl. Acad. Sci. USA 99, 12583 (2002).

[15] A.-L. Barabási and R. Albert, Science 286, 509 (1999).

[16] K.-I. Goh, B. Kahng, and D. Kim, J. Korean Phys. Soc. 46, 551 (2005).

[17] D.-H. Kim, J. D. Noh, and H. Jeong, Phys. Rev. E 70, 046126 (2004).

[18] N. Lemke, F. Herédia, C. K. Barcellos, A. N. dos Reis, and J. C. M. Momback, Bioinformatics 20, 115 (2004).

[19] C.-M. Ghim, K.-I. Goh, and B. Kahng, q-bio.MN/0411006

[20] J.-H. Kim, K.-I. Goh, B. Kahng, and D. Kim, Phys. Rev. Lett. 91, 058701 (2003).

[21] L. Zhao, K. Park, and Y.-C. Lai, Phys. Rev. E 70, 035101(R) (2004). 